\title{
Increased frequency of pneumothorax and pneumomediastinum in COVID-19 patients admitted in the ICU: A multicentre study from Mumbai, India
}

\author{
Authors: Zarir F Udwadia, ${ }^{\mathrm{A}}$ Kedar K Toraskar, ${ }^{\mathrm{B}}$ Lancelot Pinto, ${ }^{\mathrm{A}}$ Jai Mullerpatan, ${ }^{\mathrm{A}}$ Haresh D Wagh, ${ }^{\mathrm{C}}$ \\ Joanne M Mascarenhas, ${ }^{\mathrm{D}}$ Bhavesh M Gandhi, ${ }^{\mathrm{D}}$ Awatansh Tripathi, ${ }^{\mathrm{E}}$ Ayesha Sunavala, ${ }^{\mathrm{F}}$ Umang Agrawal, ${ }^{\mathrm{G}}$ \\ Viral Nanda, ${ }^{\mathrm{E}}$ Nikita Abraham, ${ }^{\mathrm{E}}$ Bony Francis, ${ }^{\mathrm{E}}$ Ravindra R Zore, ${ }^{\mathrm{H}}$ Gurudas Pundpal, ${ }^{\mathrm{H}}$ Bhoosan Gondse ${ }^{\mathrm{H}}$ and \\ Gaurav A Gupta ${ }^{\mathrm{H}}$
}

\section{Background}

There are limited data regarding the incidence of pneumothorax in COVID-19 patients as well as the impact of the same on patient outcomes.

\section{Methods}

A retrospective review of the medical records at three large tertiary care hospitals in Mumbai was performed to identify patients hospitalised with COVID-19 from March 2020 to October 2020. The presence of pneumothorax and/ or pneumomediastinum was noted when chest radiographs or CT scans were performed. Demographic and clinical characteristics of patients who developed air leak were recorded.

\section{Results}

4,906 patients with COVID-19 were admitted, with 1,324 (27\%) having severe COVID-19 disease. The overall incidence of pneumothorax and/or pneumomediastinum in patients with severe disease was $3.2 \%(42 / 1,324)$. Eighteen patients had pneumothorax, 16 had pneumomediastinum and 8 patients had both. Fourteen patients (33.3\%) developed this complication breathing spontaneously, 28 patients (66.6\%) developed it during mechanical ventilation. Overall mortality in this cohort was $74 \%$, compared with $17 \%$ in the COVID-19 patients without pneumothorax $(\mathrm{p}<0.001)$.

Authors: ${ }^{\text {A }}$ consultant chest physician, PD Hinduja National

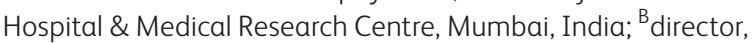
Wockhardt Hospital, Mumbai, India; ${ }^{C}$ clinical assistant, PD Hinduja National Hospital \& Medical Research Centre, Mumbai, India: ${ }^{D}$ consultant physician and intensivist, Breach Candy Hospital Trust, Mumbai, India; Etrainee, PD Hinduja National Hospital \& Medical Research Centre, Mumbai, India; ${ }^{\text {F }}$ consultant, PD Hinduja National Hospital \& Medical Research Centre, Mumbai, India; Gunior consultant, PD Hinduja National Hospital \& Medical Research Centre, Mumbai, India; ${ }^{H}$ consultant critical, Wockhardt Hospital, Mumbai, India

\section{Conclusions}

Our study demonstrates that air leaks occur with a higher frequency in patients with COVID-19 than in other ICU patients. When present, such air leaks contributed to poor outcomes with almost $74 \%$ mortality rates in these patients.

KEYWORDS: pneumothorax, pneumomediastinum, air leak in COVID-19, barotrauma in COVID-19

DOI: $10.7861 /$ clinmed.2021-0220

\section{Introduction}

Since the first case of Covid-19 was reported around 2 years ago there have been over 230 million cases worldwide and over 34 million cases in India. Studies have shown that the incidence of pneumothorax in patients with COVID-19 is about $1 \%$ in hospitalised patients and $2 \%$ in those needing intensive care. ${ }^{1-3}$ While pneumothorax has been reported in other related coronavirus pneumonia, like SARS-CoV-1 (severe acute respiratory syndrome corona virus-1) ${ }^{4}$ and MERS-CoV (Middle East respiratory syndrome coronavirus), 5 most physicians treating patients with COVID-19 pneumonia caused by SARS-COV-2 see a higher incidence of pneumothorax in these patients.

A detailed literature search we performed revealed over 200 published reports on COVID-19-associated pneumothorax/ pneumomediastinum as of June 2021, with the majority of them being case reports and small case series. There may be a higher risk of pneumothorax among COVID-19 than other pneumonia/ acute respiratory distress syndrome (ARDS); although the cause is unclear, it is not thought to be entirely due to barotrauma.,

There is a lack of Indian data regarding the incidence of pneumothorax in COVID-19 patients as well as the impact of pneumothorax on overall outcome and mortality. We present the initial Indian data from three centres describing barotrauma in patients admitted with COVID-19. We describe the clinical and radiological characteristics of COVID-19 patients developing pneumothoraces and try to identify risk factors as well as their impact on the eventual outcome. 


\section{Methods}

The study was conducted at the PD Hinduja National Hospital and Medical Research Centre, Breach Candy Hospital Trust and Wockhardt Hospital. These are tertiary care hospitals spread across the city of Mumbai, India, and were part of a network of hospitals that offered in-patient care to patients with COVID-19 disease. A retrospective review of medical records was performed to identify patients hospitalised from March 2020 to October 2020 Consecutive patients admitted to the hospital for laboratoryconfirmed COVID-19 disease were included in the analysis. The presence of a pneumothorax or pneumomediastinum was noted when chest radiographs were performed, either as part of routine care in ICU (intensive care unit) patients, or when patients clinically worsened. Of these patients, demographic and clinical characteristics of patients who developed either pneumothorax or pneumomediastinum or both were recorded. Data were summarised using non-parametric statistics.

\section{Results}

During the study period, 4,906 patients with COVID-19 were admitted. Of these, 1,324 patients (27\%) had severe COVID-19 disease, warranting ICU care. Forty-four patients had either pneumothorax or pneumomediastinum or both. Two patients who had iatrogenic central-line insertion-associated pneumothoraces were excluded from the analysis. The demographic and clinical characteristics of these 42 patients are summarised in Table 1. The median age of the patients was 60 years (IQR 54-67), with the majority of them being male $(37 / 42,88 \%)$.

The clinical presentation of the patients and the course of their illness are summarised in Table 2. Most of the patients (37/42, $88 \%$ ) were classified as having severe COVID-19 disease. The NEWS-2 (national early warning score-2) at admission was 8 (IQR 7-9). Of the 14 patients with a pneumothorax who had a CT of the chest, the median CT severity score and CT CO-RADS (COVID-19 reporting and data system) score were 15 and 5 , respectively. Only a minority of patients with pneumothorax had pre-existing lung disease $(5 / 42,16 \%)$. Sixty-six percent had comorbidities of which the most common were hypertension (48\%), diabetes (43\%) and ischaemic heart disease (28\%).

\section{Table 1. Demographic details and comorbidities}

$\begin{array}{ll}\text { Demographic details } & \\ \text { Age (mean (range)) } & 60(39-83) \text { years } \\ \text { Sex (male: female) } & 37: 5 \\ \text { Comorbidities } & \\ \text { Hypertension } & 20(48 \%) \\ \text { Diabetes } & 18(43 \%) \\ \text { Ischaemic heart disease } & 12(28 \%) \\ \text { OSA } & 3(7 \%) \\ \text { Obesity } & 2(5 \%) \\ \text { Previous lung disease } & \\ \text { Asthma } & 3(7 \%) \\ \text { Interstitial lung disease } & 2(5 \%)\end{array}$

Table 2. Severity of COVID-19

$\begin{array}{ll}\text { Severity of COVID-19 }(\mathbf{n}(\% \mathbf{N})) & 0(0) \\ \text { Mild } & 5(12) \\ \text { Moderate } & 37(88) \\ \text { Severe } & \\ \text { Other indicators of severity of lung disease } \\ \text { (mean (range)) } \\ \text { NEWS-2 score on admission } & 8(4-16) \\ \text { CT CORAD score } & 5(4-6) \\ \text { CT severity score } & 15(9-20) \\ \text { Duration of oxygenation (HFNO/NRBM/Nasal Prongs) } & 10(2-30)\end{array}$

The overall incidence of pneumothorax and/or pneumomediastinum in these patients was $3.2 \%(42 / 1,324)$. The overall incidence of pneumothorax and/or pneumomediastinum among patients admitted to the ICU with non-COVID illness during the same period was $0.6 \%(6 / 1,062)$, which was statistically significantly lower $(p<0.001)$. Overall 18 patients had pneumothorax, 16 had pneumomediastinum and eight patients had both. The mean time to onset of pneumothorax from admission was 9 days. Fourteen patients (33.3\%) developed this complication during NIV (non-invasive ventilation)/HFNC (highflow nasal cannula) as compared to 28 patients $(66.67 \%)$ who developed it during mechanical ventilation. This was statistically significant $(p<0.05)$. The median duration of documented air leak was 6 days. Lung-protective ventilation was used in all three ICUs and the highest peak pressure and plateau pressure were a mean of 35.10 and $29.75 \mathrm{cmH}_{2} \mathrm{O}$, respectively. The mean duration of mechanical ventilation was 14 days. Among all the patients who developed air leak during NIV support the CPAP (continuous positive airway pressure) was less than $10 \mathrm{cmH}_{2} \mathrm{O}$, with mean pressure of $8 \mathrm{cmH}_{2} \mathrm{O}$. The majority of patients $(28 / 42,66 \%)$ required an intercostal drain to treat pneumothorax, while the rest were managed conservatively. The decision of chest drain insertion was left to the discretion of the treating intensivist. Only one patient needed surgical intervention with VATS (video-assisted thoracoscopic) decortication. The characteristics related to the air leak and its treatment are summarised in Table 3. All patients received systemic corticosteroids, 23 patients (55\%) received

\section{Table 3. Ventilator parameters and treatment} modalities for pneumothorax

\begin{tabular}{ll}
\hline Timing of pneumothorax & $9(0-30)$ days \\
Duration of air leak & $6(1-60)$ days \\
Duration of invasive ventilation & 14 days (2 hours to 70 days) \\
Peak pressures & $35.10(21-50)$ \\
Plateau pressure & $29.75(19-40)$ \\
Treatment measures & \\
(pneumothorax) $(\mathbf{n}(\% \mathbf{N}))$ & $14(33.3)$ \\
Observation & $28(65.11)$ \\
Intercostal drain & $1(2.4)$ \\
VATS &
\end{tabular}


Table 4. Details about treatment measures and clinical outcomes

$\begin{array}{ll}\text { Treatment measures (COVID-19) }(\mathbf{n}(\% \mathbf{N})) & \\ \text { Steroids } & 42(100) \\ \text { Tocilizumab } & 23(55) \\ \text { Remdesivir } & 26(62) \\ \text { Convalescent plasma } & 10(24) \\ \text { Clinical outcome } & \\ \text { Discharge } & 9(21.4) \\ \text { Death } & 31(74) \\ \text { Discharge against medical advice } & 2(5)\end{array}$

tocilizumab, 26 patients $(62 \%)$ received remdesivir and 10 patients $(24 \%)$ received plasma therapy. The overall mortality in this cohort was $74 \%$, compared with $17 \%$ in the COVID-19 patients without pneumothorax $(p<0.001)$. Treatment measures and outcomes are summarised in Table 4.

\section{Discussion}

The first description of pneumothorax complicating COVID-19 was in the early days of the pandemic ${ }^{9}$ and since then there have been almost 200 publications on the occurrence of air leaks in these patients. The majority are scattered case reports and small series of two to three patients. Our study looks at all ICU admissions across three large hospitals in Mumbai over 7 months. Forty-two of these 1,324 ICU admissions with COVID-19 (3.2\%) developed an air leak, manifest as pneumothorax and/ or a pneumomediastinum. This is significantly higher than the six patients of $1,062(0.6 \%)$ who developed air leaks in the nonCOVID ICUs of our hospitals over the same period. Consistent with the prior literature, our cohort had an incidence of barotrauma that was higher than what is reported for ARDS from non-viral aetiologies. The incidence did not appear to be associated with ventilatory strategies which were reasonably standardised to ARDS lung-protective strategies across all three hospitals.

The strength of our study is its size, including as it does 1,324 consecutive ICU admissions (of 4,906 hospitalised patients) over 7 months from three large hospitals across Mumbai. There are only two other comparable multicentre series. The first by Martinelli et al., ${ }^{10}$ included 71 patients from 16 hospitals across the UK. The second by Miro et al. ${ }^{11}$ reviewed the incidence of pneumothorax in 61 emergency departments (EDs) across multiple hospitals in Spain. The former series is limited by including patients based on author recall and a targeted review of databases, unlike our study which systematically looked at admission details of all ICU patients. The Spanish series looked only at ED patients. Table 5 summarises the similarities and differences between our studies and these two.

Miro's study found only 40 patients of $71,904(0.56 \%)$ ED admissions showing a pneumothorax on their admission X-ray. Thus air leaks are uncommon at initial presentation to hospitals with COVID-19. In contrast, our study which looked at more critically ill ICU patients with serial X-rays and frequent CT scans showed a much higher incidence of air leak with 42 cases in 1,324 ICU admissions giving an incidence of $3.17 \%$ in this

Table 5. Comparison of our series with two other large series of patients with pneumothorax

\begin{tabular}{|c|c|c|c|}
\hline & Udwadia et al & Martinelli et $a l^{10}$ & Miro et al ${ }^{11}$ \\
\hline Setting & 3 Mumbai ICUs & 16 UK hospitals & $\begin{array}{l}61 \text { Spanish emergency } \\
\text { departments }\end{array}$ \\
\hline $\mathbf{N}$ & 1,324 critical COVID-19 patients & Case series of 71 patients & 71,904 all COVID-19 patients \\
\hline Incidence & $42 / 1,324=3.17 \%$ & NA & $40 / 71,904=0.56 \%$ \\
\hline Age & 60 (39-83) years & $21-80$ years & $66(47-74)$ years \\
\hline Male & $37 / 42(88.09 \%)$ & $57 / 71(80 \%)$ & $29 / 40(72.5 \%)$ \\
\hline Prior lung disease & $5 / 42(12 \%)$ & $28 / 71(39.4 \%)$ & $12 / 40(30 \%)$ \\
\hline $\begin{array}{l}\text { Ventilator-associated alveolar } \\
\text { leak }\end{array}$ & Ventilator-associated 28/42 (67\%) & $\begin{array}{l}\text { Possibly ventilator-associated } \\
38 / 61(62 \%)\end{array}$ & NA \\
\hline Pneumothorax alone & $18 / 42(43 \%)$ & $54 / 71(76 \%)$ & $34 / 40(85 \%)$ \\
\hline Pneumomediastinium alone & $16 / 42(38 \%)$ & $11 / 71(15.5 \%)$ & NA \\
\hline $\begin{array}{l}\text { Pneumothorax }+ \\
\text { pneumomediastinum }\end{array}$ & $8 / 42(19 \%)$ & $6 / 71(8.5 \%)$ & $6 / 40(15 \%)$ \\
\hline \multirow[t]{4}{*}{ Treatment } & Observation 14/42 (33\%) & Observation 26/71 (36\%) & Pleural tube $29 / 40(73 \%)$ \\
\hline & Pleural drain 28/42 (66\%) & Pleural drain 43/71 (60\%) & \\
\hline & VATS $1 / 42(2 \%)$ & Surgery $1 / 71(1.4 \%)$ & \\
\hline & & Palliative $3 / 71(4.2 \%)$ & \\
\hline \multirow[t]{2}{*}{ Outcome } & Death 31/42 (73\%) & Pneumothorax $63.1 \pm 6.5 \%$ & NA \\
\hline & & $\begin{array}{l}\text { Pneumomediastinum } \\
53.0 \pm 18.7 \%\end{array}$ & \\
\hline
\end{tabular}


patient population. All three series demonstrated striking male preponderance. About a third of patients in the Spanish and UK series had a history of pre-existing lung disease, unlike our patients, only $12 \%$ of whom had a prior history of lung disease. None of our 42 patients were smokers or reported a history of pneumothorax and only five had a history of pre-existing lung disease (three with well-controlled asthma and two with interstitial lung disease) which could have possibly contributed to their air leak.

Despite prompt diagnosis (due to daily serial X-rays), the mortality in our patients was high at $74 \%$ with 31 of these 42 patients succumbing to their disease. This contrasts with the overall mortality of $17 \%$ (226 deaths out of 1,282 ) in those COVID-19 patients in the ICU without an air leak. In the series of COVID-19 patients with a pneumothorax from Martinelli, similar high mortality of $63 \%$ was reported as well. Thus the occurrence of an air leak in a patient with COVID-19 pneumonia is an adverse prognostic marker signifying bad outcomes and associated with significantly higher mortality.

Interestingly our study, like that of Martinelli, ${ }^{10}$ showed that about one-third (33.3\%) of air leaks occurred in patients who never received invasive ventilation. This begs the question of why COVID-19 patients are more prone to develop air leaks and secondary pneumothoraces than other ICU patients. The typical pathology of COVID-19 pneumonia and ARDS may help explain the increased frequency of occurrence. CT scans demonstrate the peripheral distribution of the initial ground-glass opacity (GGO) changes encountered in COVID-19. ${ }^{12,13}$ The subsequent fibrosis in some of these patients have been documented to progress to is also peripheral in its distribution. ${ }^{14}$ Cyst formation and bullae in areas of airspace disease have also been noted in several radiological ${ }^{15-17}$ and autopsy studies of COVID-19. ${ }^{18,19}$ It is possible that these peripherally located bullae could rupture, either spontaneously or as a consequence of the positive pressure ventilation which the more severely ill patients with COVID-19 pneumonia are subject to. Thus while mechanical ventilation may certainly be contributing, it is not the sole risk factor, with significant numbers of air leaks occurring in patients who had never received ventilatory support (either invasive or non-invasive) at the time of their pneumothorax. This is possibly facilitated by the use of systemic corticosteroids, which is the standard of care in hypoxemic patients with COVID- $19,{ }^{20}$ and may have contributed to a lowered immune response, vulnerability to injury, and impaired healing. ${ }^{21,22}$ In keeping with this observation, there are other reports of air leaks in patients of COVID-19 pneumonia and ARDS who were not receiving mechanical ventilation at the time. $^{23,24}$

The early diagnosis of air leaks is essential and the strength of our study was that since these were all ICU patients, a chest radiograph was performed on a near-daily basis. This helped facilitate the prompt diagnosis of air leaks. Our patients developed their air leak a mean of 9 days (1-60 days) after admission to the ICU and these air leaks persisted for a mean of 6 days (1-60 days). A high index of suspicion is required to diagnose this condition. Obvious pneumothorax is generally easy to diagnose on a chest radiograph but a CT scan is more accurate in early cases of suspected pneumothorax and pneumomediastinum. Significant numbers of our patients ( 16 of $42,38 \%$ ) had pneumomediastinum in the absence of any obvious pneumothorax which may be more subtle and difficult to diagnose. This was twice the frequency noted in the UK series and maybe because unlike the patients in this series where the diagnosis of air leak was often based on physician recall and record review, diagnosis in our patients was based on daily radiographs and therefore picked up more frequently.

Prompt treatment is also crucial. Sixty-six percent of our patients required insertion of an intercostal drain at the bedside while the remainder needed careful observation without need for a drain. One patient who had a large pneumothorax and persistent air leak over 60 days underwent successful surgical repair (VATS) and was eventually discharged home. He remains well and free of recurrence highlighting the importance of surgical intervention in selected patients with persisting air leaks.

Limitation of our study was its retrospective nature with review of records at the end of 7 months period. Air leak was not actively screened for but detected as a part of regular (near-daily) chest radiograms performed as part of routine ICU protocol. Also, treatment varied across the three ICUs though most patients received drugs that were standard of care at the time of the study.

In conclusion, our study demonstrates that air leaks occur with a higher frequency in patients with COVID-19 than in other ICU patients. The incidence does not appear to be related to ventilatory strategies, as lung-protective ventilation was standard of care in all three ICUs. When present, such air leaks contributed to poor outcomes with almost $74 \%$ mortality rates in these patients which are significantly higher than the $17 \%$ mortality in patients in our other COVID-19 ICU patients without air leaks. While pneumothoraces are the commonest form of air leak, pneumomediastinum in the absence of pneumothorax occurs in $38 \%$ of patients. Air leaks are more likely to occur in patients being mechanically ventilated but a third of all air leaks occur in patients breathing spontaneously. A high index of suspicion is needed to make the diagnosis, with an air leak being considered in the differential diagnosis of any COVID-19 patient who develops sudden respiratory distress and hypoxia.

\section{Summary}

\section{What is known?}

Smaller studies and scattered case reports have shown a higher incidence of air leaks (pneumothorax and pneumomediastinum) in COVID-19 patients.

\section{What was the question?}

1) To determine the incidence of air leaks in a large cohort of patients with severe COVID-19 admitted across three ICUs over 7 months.

2) Does the occurrence of air leaks in these patients correlate with adverse outcomes and increased mortality?

\section{What was found?}

The incidence of air leaks in patients with severe COVID-19 is $3.2 \%$ which is higher than in previously reported studies. Air leak in these patients correlates with poor outcomes and carries a mortality of $74 \%$.

\section{Implications for practice?}

Air leak should be considered in the differential diagnosis of any hypoxic COVID-19 patient admitted in the ICU. They should be actively screened for and promptly treated to decrease their impact on mortality. 


\section{References}

1 Yang $X, Y u$ Y, Xu J et al. Clinical course and outcomes of critically ill patients with SARS-CoV-2 pneumonia in Wuhan, China: a singlecentered, retrospective, observational study. Lancet Respir Med 2020;8:475-81.

2 Chen N, Zhou M, Dong X et al. Epidemiological and clinical characteristics of 99 cases of 2019 novel coronavirus pneumonia in Wuhan, China: a descriptive study. Lancet 2020;395:507-13.

3 Yang F, Shi S, Zhu J, et al. Analysis of 92 deceased patients with COVID-19. J Med Virol 2020;92:2511-5.

4 Sihoe ADL, Wong RHL, Lee ATH et al. Severe acute respiratory syndrome complicated by spontaneous pneumothorax. Chest 2004;125:2345-51.

5 Al-Dorzi HM, Alsolamy S, Arabi YM. Critically ill patients with Middle East respiratory syndrome coronavirus infection. Critical Care 2016;20:65.

6 Das KM, Lee EY, Al Jawder SE et al. Acute Middle East respiratory syndrome coronavirus: temporal lung changes observed on the chest radiographs of 55 patients. Am J Roentgenol 2015;205:W267-74.

7 Lemmers DHL, Abu Hilal M, Bnà C et al. Pneumomediastinum and subcutaneous emphysema in COVID-19: barotrauma or lung frailty? ERJ Open Res 2020;6:00385-2020.

8 McGuinness $\mathrm{G}$, Zhan C, Rosenberg $\mathrm{N}$ et al. Increased incidence of barotrauma in patients with COVID-19 on invasive mechanical ventilation. Radiology 2020;297:E252-62.

9 Sun R, Liu H, Wang X. Mediastinal emphysema, giant bulla, and pneumothorax developed during the course of COVID-19 pneumonia. Korean J Radiol 2020;21:541.

10 Martinelli AW, Ingle T, Newman ] et al. COVID-19 and pneumothorax: a multicentre retrospective case series. Eur Respir ] 2020;56:2002697.

11 Miró Ò, Llorens P, Jiménez S et al. Frequency, risk factors, clinical characteristics, and outcomes of spontaneous pneumothorax in patients with coronavirus disease 2019: a case-control, emergency medicine-based multicenter study. Chest 2020;159:1241-55.

12 Smith DL, Grenier J-P, Batte C, Spieler B. A characteristic chest radiographic pattern in the setting of the COVID-19 pandemic. Radiol Cardiothorac Imaging 2020;2:e200280.
13 Altmayer S, Zanon M, Pacini GS et al. Comparison of the computed tomography findings in COVID-19 and other viral pneumonia in immunocompetent adults: a systematic review and meta-analysis. Eur Radiol 2020;30:6485-96.

14 Udwadia Z, Pokhariyal P, Tripathi AR, Kohli A. Fibrotic interstitial lung disease occurring as sequelae of COVID-19 pneumonia despite concomitant steroids. Lung India. 2021;38:S61-3.

15 Martinelli AW, Ingle T, Newman J et al. COVID-19 and pneumothorax: a multicentre retrospective case series. Eur Respir ] 2020;56:2002697.

16 Sun R, Liu H, Wang X. Mediastinal emphysema, giant bulla, and pneumothorax developed during the course of COVID-19 pneumonia. Korean J Radiol 2020;21:541.

17 Liu K, Zeng Y, Xie P et al. COVID-19 with cystic features on computed tomography. Medicine (Baltimore) 2020;99:e20175.

18 Bharat A, Querrey M, Markov NS et al. Lung transplantation for patients with severe COVID-19. Sci TransI Med 2020;12:eabe4282. https://www.worldometers.

19 Schwensen HF, Borreschmidt LK, Storgaard M, et al. Fatal pulmonary fibrosis: a post-COVID-19 autopsy case. J Clin Pathol 74:1-3.

20 Horby P, Lim WS, Emberson JR et al. Dexamethasone in hospitalized patients with Covid-19 - preliminary report. N Engl J Med 2020384:693-704.

21 van Schaik E, Ramnarain D, Pouwels S. Pneumothorax due to a non-traumatic thoracic wall rupture due to steroid induced muscle wasting. Ann Thorac Surg 2021;:S0003-4975.

22 Segarra FO, Sherman DS. Spontaneous pneumothorax during steroid therapy in pulmonary tuberculosis. Dis Chest 1962;41:354-5.

23 Goldman N, Ketheeswaran B, Wilson H. COVID-19-associated pneumomediastinum. Clin Med J R Coll Physicians London 2020;20:91-3.

24 Zantah M, Dominguez Castillo E, Townsend R, Dikengil F, Criner G]. Pneumothorax in COVID-19 disease: incidence and clinical characteristics. Respir Res 2020;21:236.

Address for correspondence: Dr Zarir Udwadia, PD Hinduja National Hospital and Medical Research Centre, Mumbai 400016, India.

Email: zfu@hindujahospital.com 